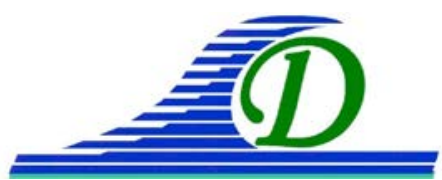

XIII İmes Journées Nationales Génie Côtier - Génie Civil

Dunkerque, 2-4 juillet 2014

DOI:10.5150/jngcgc.2014.058 C Editions Paralia CFL

disponible en ligne - http://www.paralia.fr - available online

\title{
Auscultation d'ouvrages de protection en remblai par capteur composite géotextile à fibre optique
}

\author{
Olivier ARTIERES ${ }^{1}$
}

\author{
1. TenCate Geosynthetics, \\ 9 rue Marcel Paul, BP 40080, 95873 Bezons Cedex, France. \\ o.artieres@tencate.com
}

\section{Résumé :}

La mesure des déformations dans un ouvrage en remblai ou en enrochements est maintenant accessible avec la technologie des capteurs à fibres optiques, qui associée aux propriétés des géotextiles, comme par exemple leur frottement d'interface, permet un bon transfert des mouvements du sol vers le capteur. Ils ont une sensibilité élevée, inférieure à $0,01 \%$ en déformation et une précision de $0,1^{\circ} \mathrm{C}$ en température, permettant de détecter et de localiser avec une résolution spatiale de l'ordre du mètre des déplacements d'enrochements ou des instabilités, mais aussi des fuites. Ils sont polyvalents tant pour ausculter des ouvrages locaux, comme des épis, que des infrastructures de plusieurs dizaines de kilomètres, comme les digues de protection. Plusieurs dizaines d’ouvrages sont maintenant auscultés depuis plus de 10 ans.

Mots-clés : Auscultation, Alerte, Détection, Maintenance, Surveillance, Fibre optique, Déformation, Température, Instabilité, Mouvement de sol, Digue, Géotextile, Enrochement, Capteur.

\section{Abstract:}

Strain in earthworks is now easier to measure by using fibre optic sensing technologies combined with geotextile properties, such as very good soil friction interface enhancing the transfer of soil displacement to the sensors. They have high strain sensitiveness below $0.01 \%$ and a temperature accuracy of $0.1{ }^{\circ} \mathrm{C}$, combined with a high spatial resolution in the range of $1 \mathrm{~m}$. They are used to monitor either local structures such as groynes, or long infrastructures of several tenths of kilometres such as protection dikes, with the same accuracy. Several tenths of structures are now monitored globally with this solution for more than 10 years demonstrating its durability.

Keywords: Monitoring, Warning Maintenance, Surveillance, Fibre optic, Strain, Temperature, Instability, Soil movement, Dike, Geotextile, Stones, Sensor.

\section{Introduction}

L'auscultation des digues de protection fait partie intégrante de leur plan de surveillance et de maintenance. Elle peut également être intégrée à un système d'alerte précoce en cas de sollicitations extrêmes, comme par exemple lors de tempêtes. Les digues de protection peuvent couvrir plusieurs centaines de mètres à plusieurs kilomètres. On doit 
pouvoir les ausculter avec une résolution spatiale suffisante pour ne pas manquer un évènement préjudiciable, comme une érosion de pied, des affaissements ou des glissements, pour générer des alertes précoces face à des évènement soudains.

La mesure distribuée par fibre optique permet d'ausculter un ou plusieurs ouvrages simultanés en conservant une précision de mesure et une résolution spatiale de même qualité que les capteurs ponctuels conventionnels. Cette base technologique associée aux propriétés mécaniques et hydrauliques des géotextiles a permis de concevoir une solution performante pour l'auscultation des ouvrages hydrauliques en remblai.

La performance de cette solution a été validée sur plusieurs sites expérimentaux à l'échelle 1 (ARTIERES et al., 2010). Ce dispositif est également en place depuis 2006 sur de nombreuses digues. Après un descriptif de la technologie du capteur, son utilisation dans les cas d'une instabilité de talus amont de digue soumise à la marée et du mouvement d'enrochements d'une digue de protection seront présentés.

\section{La solution d'auscultation à base du capteur géotextile à fibre optique}

\subsection{La technologie de mesure par fibre optique}

Les capteurs à fibre optique ont été largement utilisés depuis plusieurs années dans les applications de génie civil, en particulier pour la surveillance des conduites d'hydrocarbure, dans des systèmes de surveillance de l'état de structure, ou des applications hydrauliques comme des barrages ou des digues en béton ou en terre. En associant des fibres optiques sur un géotextile (figure 1), TenCate GeoDetect ${ }^{\circledR}$ est un capteur géotextile innovant qui améliore les performances des fibres optiques lorsqu'elles sont utilisées en contact avec des matériaux granulaires, sol ou enrochements: le géotextile créé une excellente interface d'ancrage avec le milieu environnant. Grâce à la bonne liaison des câbles optiques sur le géotextile, de très faibles déformations du sol peuvent être détectées.

Différentes technologies de capteurs par fibre optique peuvent être utilisées : les réseaux de Bragg (FBG) employés pour le suivi de sections d'ouvrages, ou la mesure répartie, plus courante dans les ouvrages hydrauliques et utilisée dans les études de cas aux paragraphes 3 et 4 . Les réseaux de Bragg sont des modifications locales de l'indice optique à l'intérieur de la fibre optique sous forme d'une série de petits miroirs inscrits sur une distance de quelques millimètres de long et réfléchissant une longueur d'onde donnée, proportionnelle à la température et à la déformation. A l'inverse des réseaux de Bragg qui sont des mesures ponctuelles au lieu de l'inscription des singularités, les technologies réparties Brillouin ou Raman décrites au §2.2 mesurent tout point le long de la fibre sur des distances de plusieurs dizaines de kilomètres. Ces deux technologies permettent une mesure très précise de paramètres comme la température ou la déformation, avec des fréquences de mesure soit statiques $(<1 \mathrm{~Hz})$ soit dynamiques (de 


\section{XIII $I^{\text {èmes }}$ Journées Nationales Génie Côtier - Génie Civil \\ Dunkerque, 2-4 juillet 2014}

$1 \mathrm{~Hz}$ à $2 \mathrm{kHz}$ ). Ces solutions sont décrites plus en détail dans de précédentes publications (ARTIERES \& DORTLAND, 2011).

La solution d'auscultation comprend le capteur géotextile à fibres optiques, l'instrumentation et le logiciel d'acquisition de données (figure 1). Différentes stratégies d'auscultation peuvent être prévues lors du dimensionnement, comme une auscultation périodique ou une mesure continue à des fins d'alerte précoce.

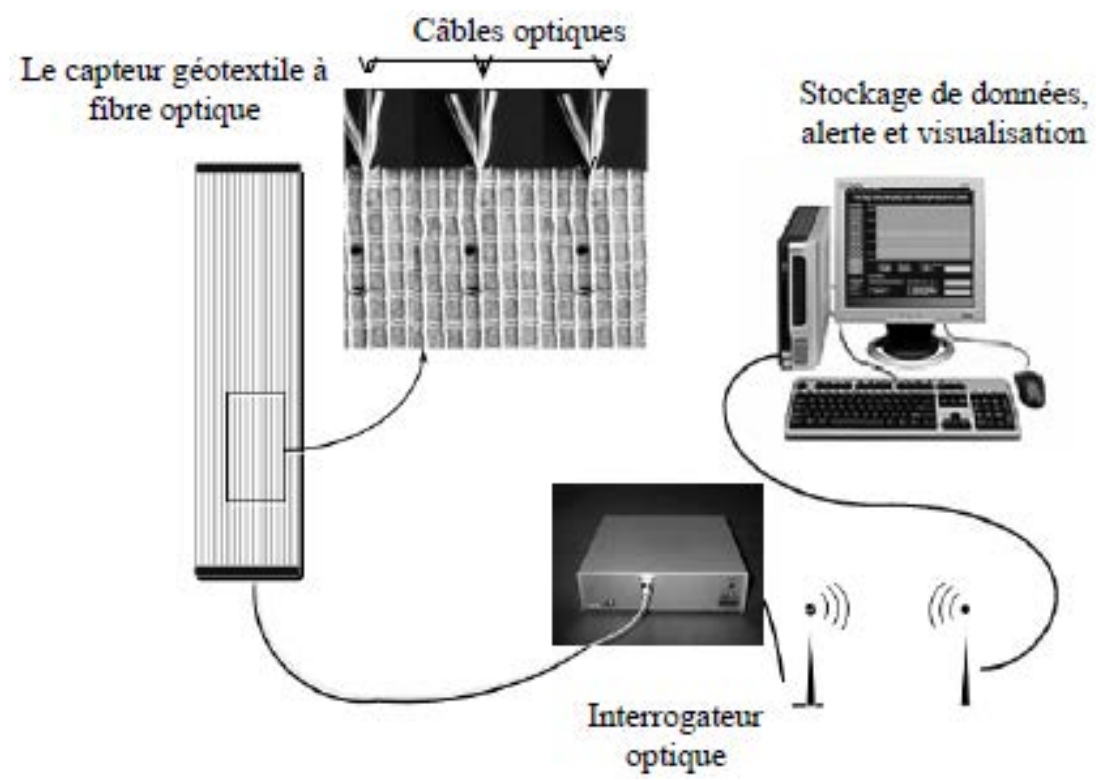

Figure 1. Composants du système d'auscultation géotextile à fibre optique.

En comparaison avec des techniques d'auscultation constituées de capteurs ponctuels câblés individuellement, cette solution mesure en continu jusqu'à plusieurs dizaines de milliers de points avec une seule instrumentation sur toute la longueur de la structure. La résolution spatiale peut être dans certains cas de 0,5 m. Le seuil de détection en déformation est inférieur à $0,01 \%$ et la précision de mesure de la température est inférieure à $0,1^{\circ} \mathrm{C}$. La technologie de mesure par fibres optiques ne nécessite aucune calibration, il n’y a pas de dérive dans le temps, car ce sont des caractéristiques intrinsèques à la fibre optique qui sont mesurées. Dans certains cas, une compensation de la température peut être nécessaire pour des variations de plus de $10^{\circ} \mathrm{C}$.

Le capteur composite geotextile porte et protège plusieurs câbles optiques redondants.

\subsection{Le capteur géotextile à fibre optique pour la mesure répartie}

Le capteur se présente sous forme de bandes géotextile de $76 \mathrm{~cm}$ de large portant 2 câbles optiques pour la mesure des déformations et deux câbles optiques pour la mesure de la température, dont deux redondants. Ces capteurs sont reliés par un câble optique de liaison de quelques dizaines de mètres à plusieurs centaines de mètres à un 
interrogateur optoélectronique Raman et/ou Brillouin. Il n’y a donc aucun appareil électromécanique ni de source électrique dans l'ouvrage.

L'interrogateur envoie un pulse laser de quelques nanosecondes qui parcourt toute la longueur de la fibre optique. En chaque point de la fibre, le pulse laser interfère avec la structure moléculaire de la matière en rétrodiffusant un spectre de lumière (figure 2) qui est analysé. La longueur d'onde des pics secondaires Brillouin dépend de l'état de déformation de la fibre optique. La résolution spatiale de la fibre est d'environ $1 \mathrm{~m}$, ce qui signifie que le système génère un point de mesure tous les mètres.

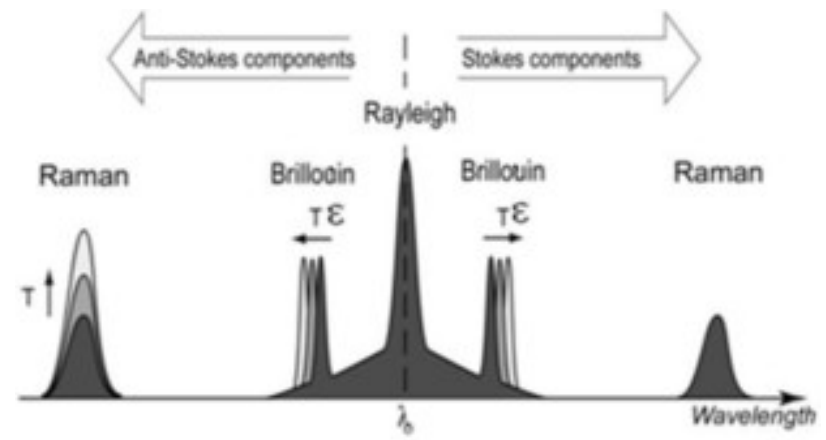

Figure 2. Spectre de lumière rétrodiffusé lors de la mesure répartie sur fibre optique ( $\lambda 0$ est la longueur d'onde de la lumière incidente).

\section{Digue urbaine maritime à Boston (Angleterre)}

\subsection{Le contexte}

Boston est une ville de la côte Est de l'Angleterre sujette à un risque élevé d'inondation. Elle est raccordée à la mer par un canal qui est soumis à un fort marnage jusqu'à $6 \mathrm{~m}$ d'amplitude au printemps dû à la marée (figure 3). Une digue en terre du centre ville à proximité de l'écluse "Grand Sluice" présente des signes d'instabilité avec des zones de glissement. Cet ouvrage géré par l' "Environment Agency" a été choisi comme tronçon de démonstration de technologies d'auscultation dans le cadre du projet européen "Urbanflood" du 7ème PCRD. Il a été instrumenté sur une longueur de 300 mètres environ par le capteur composite géotextile fibre optique pour suivre l'évolution des glissements de terrain. Ainsi, avec une résolution longitudinale de $1 \mathrm{~m}$, ce sont 600 points qui sont acquis en simultané à chaque pas de mesure.

\subsection{Installation du système d'auscultation}

Le capteur a été déroulé en continu sur deux niveaux le long du talus, permettant d'intercepter les zones de glissement sur le talus amont (figure 4). Les bandes sont placées dans des tranchées de 20 ou $40 \mathrm{~cm}$ de profondeur, afin de ne pas créer d’instabilité locale lors des travaux. Pour la même raison, l’installation est réalisée par 


\section{XIII ${ }^{\text {èmes }}$ Journées Nationales Génie Côtier - Génie Civil \\ Dunkerque, 2-4 juillet 2014}

sections successives de $20 \mathrm{~m}$ de long. Les câbles optiques sont raccordés directement à l’instrumentation placée dans un local de l'écluse situé à proximité.

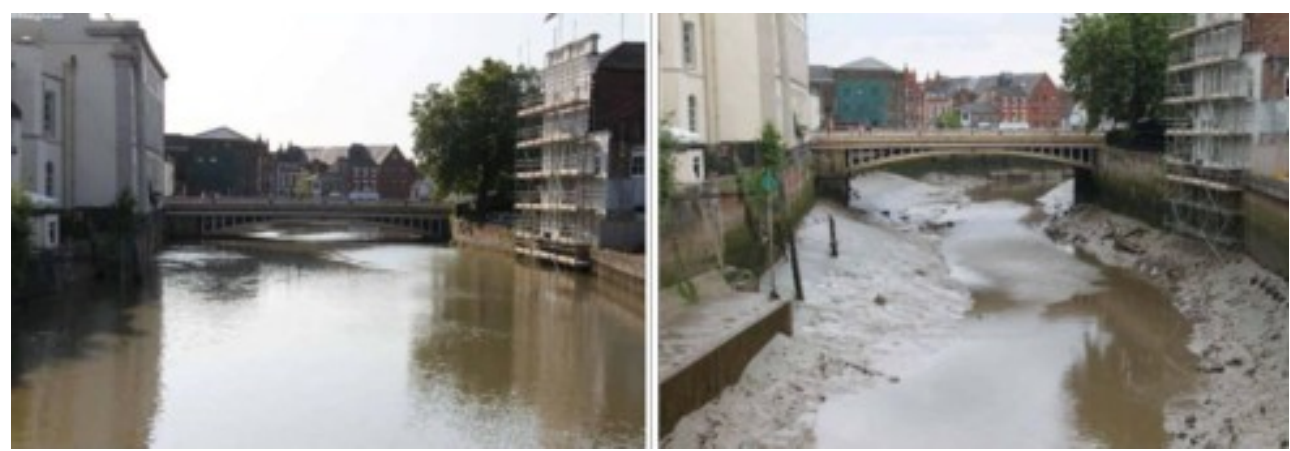

Figure 3. Le canal au centre de Boston, à marée haute et à marée basse.
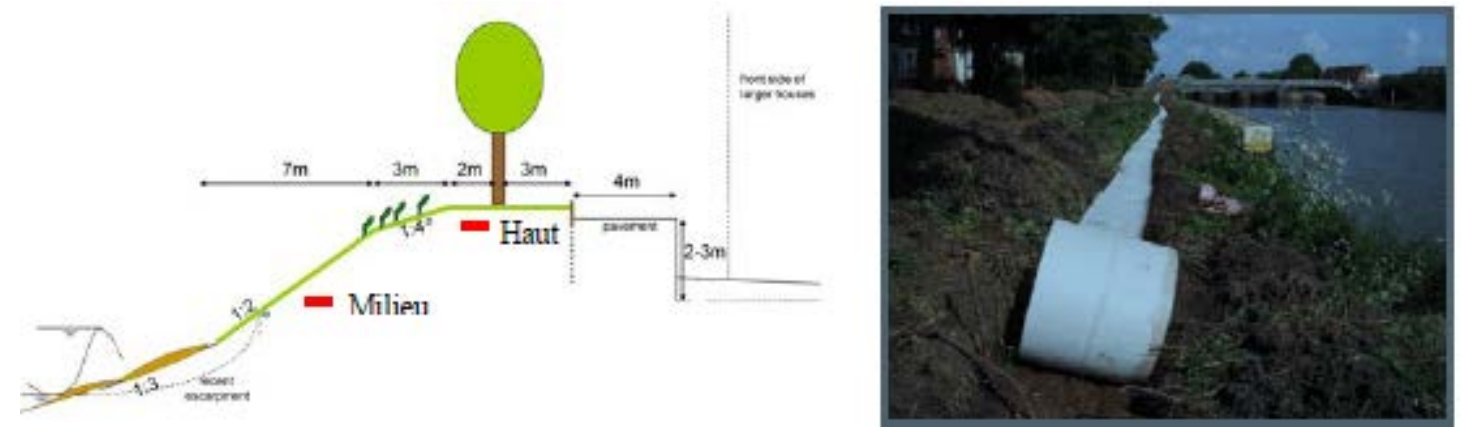

Figure 4. Coupe de la digue auscultée et position des deux niveaux de bandes capteur (en rouge à gauche) et pose de la bande en milieu de pente (à droite).

\subsection{Evolution sur une année}

Les mesures des déformations le long du capteur sont effectuées par TenCate Geosynthetics. Les données brutes sont ensuite analysées par les partenaires du projet Urbanflood. La résolution longitudinale pour ce projet est de $50 \mathrm{~cm}$. La première série de mesures a été effectuée en continu sur une période de 2 mois entre juin et aout 2011, suivi de deux mesures instantanées en avril et novembre 2012.

L'évolution des déformations relatives le long de l'ouvrage est en moyenne assez faible, entre +500 et -1000 microdéformations, soit entre $+0,05 \%$ et $-0,1 \%$. La partie haute (figure 5) est logiquement la plus sollicitée. Quelques sections indiquent des tractions très localisées à 90,110, 167 et $230 \mathrm{~m}$ avec des déformations supérieures à $0,1 \%$. L’ouvrage reste néanmoins stable, sans zone de rupture évolutive. 


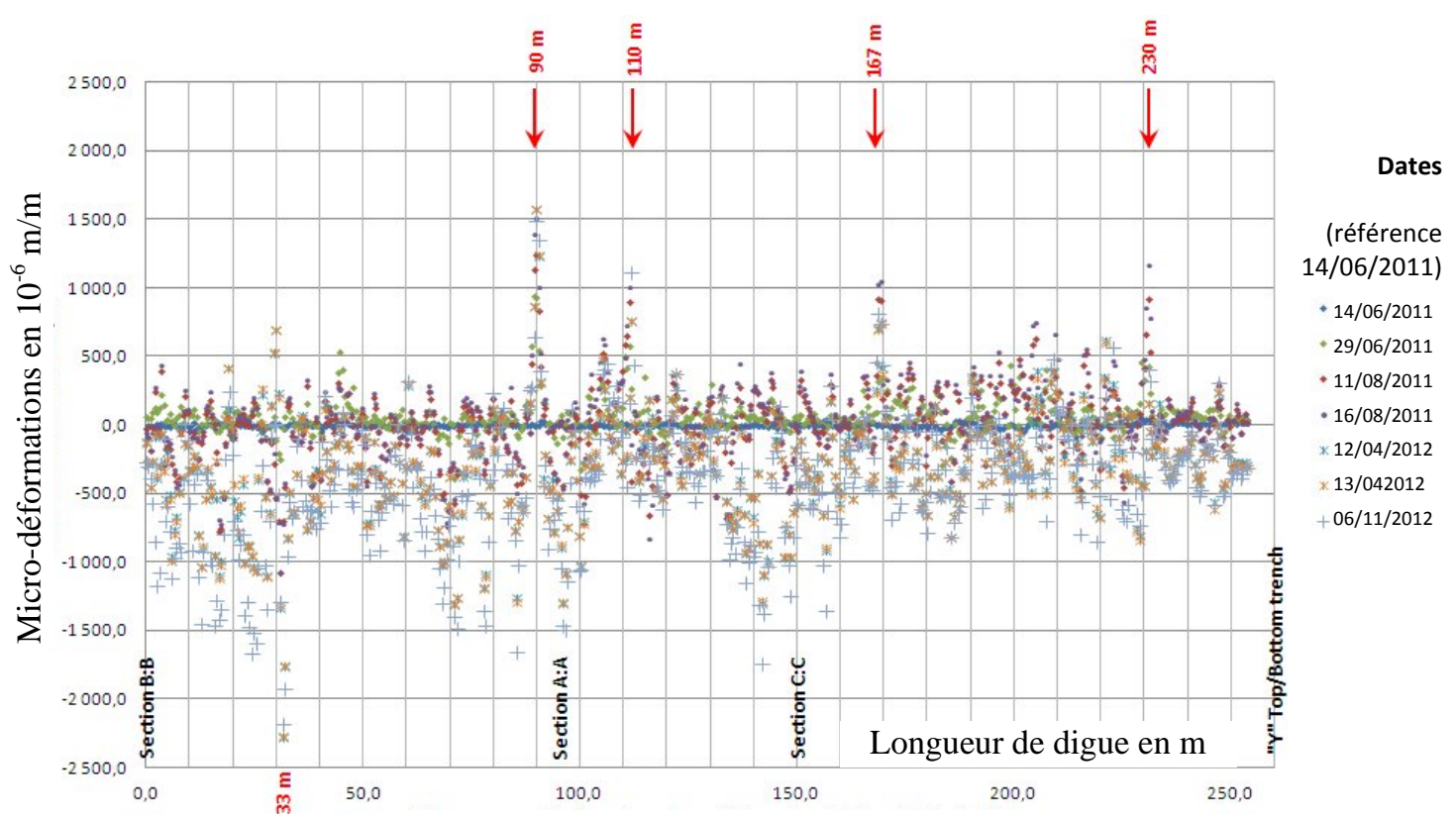

Figure 5. Déformations relatives le long de la digue de Boston - Bande supérieure

\section{Digue de protection sur le fleuve jaune (Chine)}

\subsection{Le fleuve jaune}

Avec ses 5464 km de long, le fleuve jaune (Huang He) est le deuxième plus long fleuve de Chine. Son nom lui vient de sa forte turbidité, car il charrie de grandes quantités d'alluvions qui fertilisent la grande plaine du Nord de la Chine. Il possède un lit majeur très large, de plusieurs kilomètres, et un lit mineur très changeant. Sa période d'étiage est hivernale et de plus en plus longue à cause des nombreux prélèvements dont il fait l'objet pour les besoins agricoles. Néanmoins ses crues estivales peuvent être fortes. Des digues de protection et des éperons ont été construits sur son parcours, notamment dans la province de Henan, non loin de Zhengzhou. Le "Yellow River Conservancy Commission" (YRCC) constate une forte érosion du talus amont des digues de protection en période de crue (figure 6) et a souhaité ausculter un éperon témoin au cours de la saison estivale 2012.
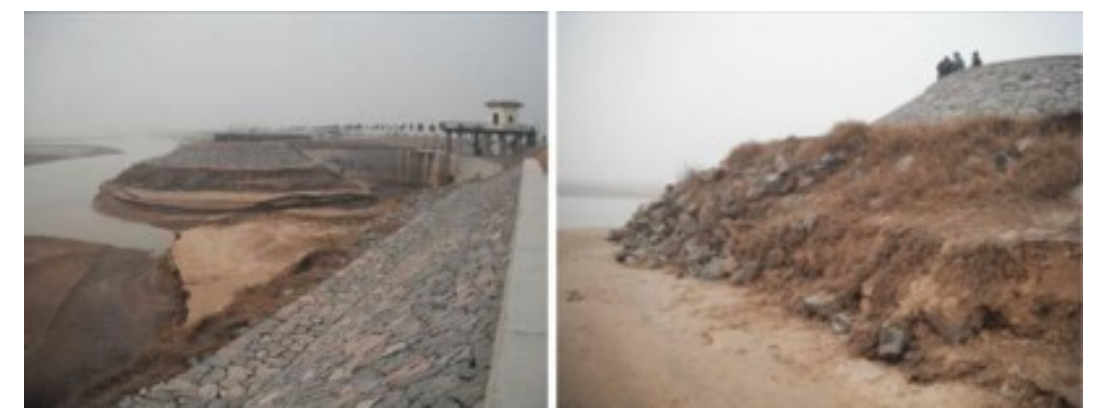

Figure 6. Eperon de la digue de protection montrant une forte érosion externe en pied. 


\section{XIII $I^{\text {èmes }}$ Journées Nationales Génie Côtier - Génie Civil \\ Dunkerque, 2-4 juillet 2014}

\subsection{Conception et installation}

L'objectif de la mesure est le suivi du mouvement du revêtement en enrochements lors d'une crue. Une longueur d'environ 120 m a été équipée d'une bande capteur géotextile à fibres optiques sous la ligne des plus hautes eaux observées. Cette longueur couvre la partie exposée de l'éperon ainsi que l'anse de digue principale entre deux éperons successifs (figure 7). La bande capteur a été déroulée sur une plateforme entre deux géotextiles non-tissés épais de protection, l'ensemble étant recouvert des enrochements. Sur une longueur de $10 \mathrm{~m}$, des "geobags", sacs géotextile remplis de sol, ont été substitués aux enrochements. Leur dimension est de $1,4 \mathrm{~m} \times 1,8 \mathrm{~m}$.

La solution de détection et d'alerte à été installée fin mai 2012. Le repérage de la distance mesurée le long de la bande capteur par rapport à sa position dans l'ouvrage est indiquée en figure 9. La bande capteur sur le site est reliée à l'instrumentation placée dans un local climatisé distant de $300 \mathrm{~m}$. Reliée à internet, celle-ci est consultable et paramétrable à distance depuis la France. Elle génère une mesure toutes les heures, cette fréquence pouvant être resserrée à une dizaine de minutes si nécessaire.
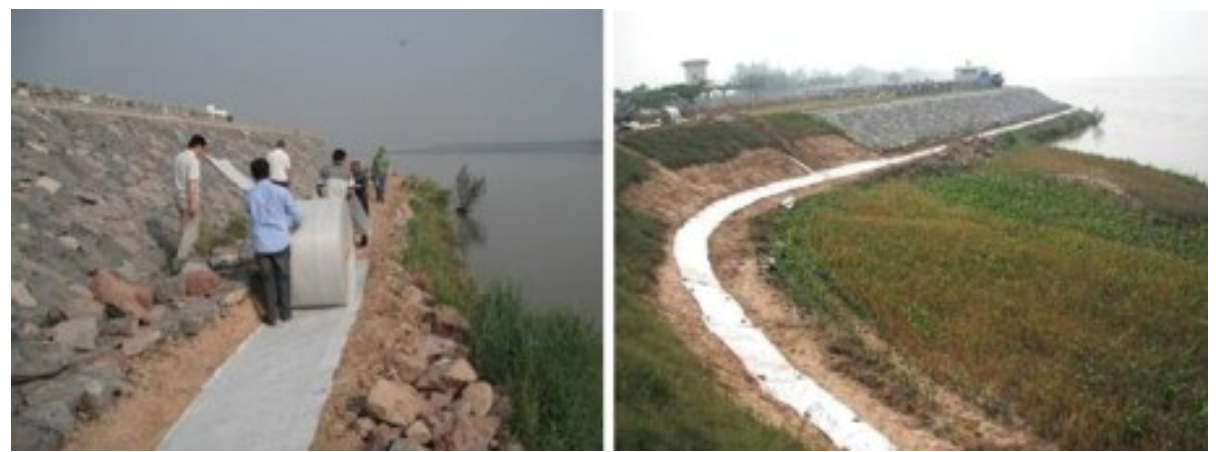

Figure 7. Pose de la bande capteur composite sur la berge intermédiaire.

\subsection{Mesures}

Le YRCC a créé une crue artificielle le 19 Juin en baissant le niveau du barrage Xiaolangdi en amont, afin de créer une onde de crue d'environ 3 mètres. Les premiers mouvements d'enrochement sur l'éperon ausculté sont détectés à partir du 22 juin par des modifications de déformations sur la bande capteur (figure 8) entre les abscisses 60 et $80 \mathrm{~m}$, et vers $100 \mathrm{~m}$, avec un pic de déformation à $500 \mu \varepsilon$.

Les mouvements principaux apparaissent entre le 24 et le 26 juin avec l'arrivée du niveau d'eau maximum. On constate des zones présentant des mouvements importants supérieurs à $1000 \mu \varepsilon$, à $50 \mathrm{~m}$, entre 60 et $90 \mathrm{~m}$, et après $100 \mathrm{~m}$. La zone entre 90 et $100 \mathrm{~m}$ ne fait pas l'objet de mouvements : c’est la partie couverte par les "geobags", car beaucoup plus stables que les enrochements d'une trentaine de kilos. La figure 9 indique la position des zones à fort mouvement. La stabilité revient après le 30 juin. 

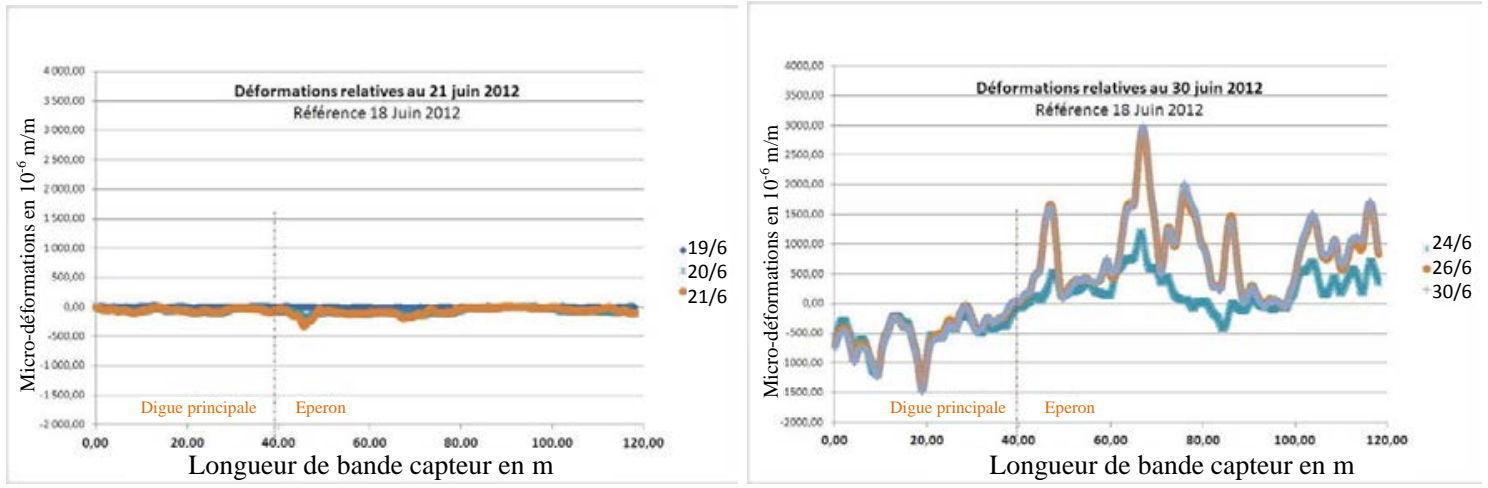

Figure 8. Déformations mesurées respectivement au 21, 23 et 30 juin.

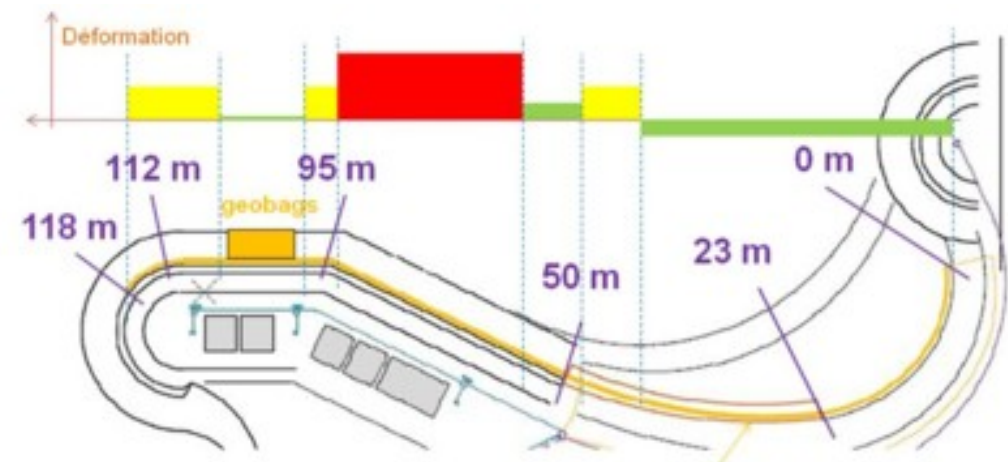

Figure 9. Position de la bande capteur et zonage des déformations mesurées le 30 juin.

\section{Conclusions}

La solution d'auscultation et d'alerte basée sur le capteur géotextile à fibres optiques permet de détecter précisément les signes précurseurs de dysfonctionnement, comme les mouvements d'une couverture en enrochement et à localiser les zones instables d'un talus de façon très précise, à moins d'un mètre près.

Cette solution contribue à réduire les risques. Elle permet la génération d'une alerte précoce en cas d'une variation inattendue des propriétés des matériaux ou des conditions locales.

\section{Références bibliographiques}

ARTIERES O., BECK Y.L., KHAN A.A., CUNAT P., FRY J.J., COURIVAUD J.R., GUIDOUX C., PINETTES P. (2010). Assessment of Dam and Dikes behaviour with a fiber optic based monitoring solution. Proc. of the 2nd Dam Maintenance Conference, Zaragoza, november 2010, pp 79-86.

ARTIERES O. DORTLAND G. (2011). Six years earthworks monitoring with a fiber optic geotextile enabled sensor. Compte-rendus du 8ème Symposium International on Field Measurements in GeoMechanics, Berlin, September 12-16, 2011. 